[white paper]

Diamond Open Access

\title{
A função módulo $|x|$ possui derivada
}

\author{
Colaboração Matemática Aberta ${ }^{1}$ \\ 1 de Novembro de 2020
}

\section{Resumo}

Nosso objetivo neste artigo é introduzir o conceito de Derivada Fraca e mostrar que a função $u(x)=|x|, x \in(-1,1)$, possui sim derivada.

palavras-chave: Derivada fraca, medida de Lebesgue, integral de Lebesgue.

A versão mais atualizada deste artigo está disponível em https://osf.io/34z8x/download

\section{Como ser coautor(a) deste artigo?}

1. Envie-nos suas observações e responda às seguintes perguntas.

2. Quais discussões você adicionaria a este artigo?

3. Você encontrou algum erro ou argumento inconsistente? Dê uma justificativa detalhada.

${ }^{1}$ Todos os autores com suas afiliações aparecem no final deste artigo. 


\section{Introdução}

4. Este artigo está baseado, principalmente, nas referências [1,2].

5. Um exercício relativamente simples do Cálculo Diferencial é mostrar que a função $u(x)=|x|, x \in(-1,1)$, não possui derivada em todo seu domínio.

6. Entretanto, a função $u$ possui derivada no sentido fraco, cuja conceituação é o principal objetivo deste artigo.

7. Por trás desse conceito, está a Integral de Lebesgue, uma das descobertas mais importantes da Análise no século passado, que estendeu notavelmente a Integral de Riemann, resolveu num período de poucos anos os problemas fundamentais da Teoria da Integração e deu um impulso relevante à Análise Funcional, à Teoria das Equações Diferenciais e Integrais e à Teoria da Probabilidade.

8. O ponto básico dessa nova teoria foi a introdução da noção de medida.

9. Destacamos que alguns conceitos serão apresentados apenas com propósito informativo. Que isso possa servir para trazer curiosidade e incentivar o leitor a se aprofundar no assunto.

\section{Definições importantes}

10. Considere $\wp(X)$ o conjunto das partes de um conjunto qualquer $X$. Um subconjunto $A$ de $\wp(X)$ é uma $\sigma$-álgebra de $X$ se:

(a) $\varnothing, X \in A$;

(b) Dado um conjunto $B \in A$, o complementar $B^{\complement}=X \backslash B \in A$;

(c) Qualquer que seja a sequência $\left(B_{n}\right)$ de elementos de $A$, a união $\cup_{n \in \mathbb{N}} B_{n} \in A$.

11. $A=\{\varnothing, X\}$ é a menor $\sigma$-álgebra de $X$ e $A=\wp(X)$ é considerada a maior $\sigma$-álgebra de $X$. 
12. Sejam $\mathbb{N}=\{1,2,3, \ldots\}$ o conjunto dos números naturais e $A$ o conjunto formado por $\varnothing,\{1,3,5, \ldots\},\{2,4,6, \ldots\}, \mathbb{N}$. Então, $A$ é uma $\sigma$-álgebra de $X$.

13. Considere $A \subset \wp(X)$, não necessariamente uma $\sigma$-álgebra, e $\sigma(A)$ definido como a interseção de todas as $\sigma$-álgebras que contém $A$. Esta é chamada de $\sigma$-álgebra gerada por $A$.

14. A $\sigma$-álgebra gerada pela família de intervalos abertos de $\mathbb{R}$ é conhecida como a $\sigma$-álgebra de Borel, e seus elementos são chamados de borelianos. Denotaremos tal $\sigma$-álgebra por $\mathcal{B}$.

15. Tem-se que $\left\{a_{1}, a_{2}, \cdots, a_{n}\right\} \in \mathcal{B}$ e $I \in \mathcal{B}$, em que $n \in \mathbb{N}, a_{i}^{\prime} s \in \mathbb{R}$ e $I \subset \mathbb{R}$ é um intervalo qualquer.

16. Seja $A$ uma $\sigma$-álgebra de um conjuto não vazio $X$. O par $(X, A)$ é dito um espaço mensurável e os conjuntos em $A$ são os conjuntos mensuráveis.

17. Por (15), qualquer intervalo $I \subset \mathbb{R}$ é mensurável se considerarmos o $\operatorname{par}(\mathbb{R}, \mathcal{B})$.

18. Dizemos que $f: X \rightarrow \mathbb{R} \cup\{+\infty,-\infty\}$ é $A$-mensurável ou mensurável se, $\forall \delta \in \mathbb{R}$, o conjunto $\left.\left.[f>\delta]=\{x \in X: f(x)>\delta\}=f^{-1}(] \delta,+\infty\right]\right)$ é mensurável, isto é, se $[f>\delta] \in A$.

19. No espaço $(\mathbb{R}, \mathcal{B})$, as funções contínuas de $\mathbb{R}$ em $\mathbb{R}$ são mensuráveis, $\operatorname{como} \operatorname{sen}(x), \cos (x), x^{2}, \frac{x}{x^{2}+1}$.

\section{Medida em uma $\sigma$-álgebra}

20. Seja $A$ uma $\sigma$-álgebra. Chama-se medida em $A$ uma função $\mu: A \rightarrow$ $[0,+\infty]$ que satisfaz:

(a) $\mu(\varnothing)=0$; 
(b) Se $\left(A_{n}\right)_{n \in \mathbb{N}}$ é uma sequência de conjuntos dois a dois disjuntos em $A$, então

$$
\mu\left(\bigcup_{n \in \mathbb{N}} A_{n}\right)=\sum_{n=1}^{+\infty} \mu\left(A_{n}\right) .
$$

21. Entendemos aqui que $a+\infty=+\infty+a=+\infty$ para todo $a \in \mathbb{R}$.

22. Sejam $X$ um conjunto e $A$ uma $\sigma$-álgebra de $X$. Considere a função $\mu: A \rightarrow[0,+\infty]$ dada por

$$
\mu(B)=\left\{\begin{array}{cl}
\text { número de elementos de } \mathrm{B}, & \text { se } B \text { é finito } \\
+\infty, & \text { se } B \text { é infinito }
\end{array} .\right.
$$

Temos que $\mu$ é a medida caracterizada como medida de contagem.

23. Do Lema $3.3 \mathrm{em}[2]$, segue que, se $E, F \in A$ e $\mu$ é uma medida em $A$, então $E \subseteq F \Rightarrow \mu(E) \leq \mu(F)$.

24. Considere $X=\mathbb{R}$ e $A=\mathcal{B}$ a $\sigma$-álgebra de Borel. Existe uma única medida $\mu$ definida em $\mathcal{B}$ tal que $\mu(a, b)=b-a$, para todo intervalo aberto $(a, b)$. Essa medida recebe o nome de medida de Lebesgue. Essa afirmação pode ser vista no Exemplo 3.2-d) em [2].

25. A medida de Lebesgue é a medida com a qual define-se o conceito de Integral de Lebesgue e, em seguida, de derivada fraca.

26. Como exercício, vamos mostrar que, se $P=\left\{a_{1}, \cdots, a_{n}\right\} \in \mathcal{B}, n \in \mathbb{N}$, então

$$
\mu(P)=0 .
$$

27. Vamos mostrar que a medida de Lebesgue de um conjunto formado por um único ponto é 0 , isto é, dado $b_{1} \in \mathbb{R}$,

$$
\mu\left(\left\{b_{1}\right\}\right)=0 .
$$

28. Não é difícil mostrar que $\left\{b_{1}\right\}=\bigcap_{n=1}^{+\infty}\left(b_{1}-\frac{1}{n}, b_{1}+\frac{1}{n}\right)$. 
29. Portanto, a medida do ponto é igual a medida da interseção, ou seja,

$$
\mu\left(\left\{b_{1}\right\}\right)=\mu\left(\bigcap_{n=1}^{+\infty}\left(b_{1}-\frac{1}{n}, b_{1}+\frac{1}{n}\right)\right) .
$$

30. Além disso, como a interseção está contida em todos os intervalos $\left(b_{1}-\frac{1}{n}, b_{1}+\frac{1}{n}\right), n \in \mathbb{N}$, segue de 23 , 24) e 29) que, para todo $n \in \mathbb{N}$,

$$
\mu\left(\left\{b_{1}\right\}\right) \leq \mu\left(b_{1}-\frac{1}{n}, b_{1}+\frac{1}{n}\right)=\frac{2}{n} .
$$

31. Podemos utilizar o Teorema do Confronto [3, Teorema 6] obter

$$
0 \leq \mu\left(\left\{b_{1}\right\}\right) \leq \frac{2}{n}, \forall n \in \mathbb{N} \Rightarrow \lim _{n \rightarrow \infty} 0 \leq \lim _{n \rightarrow \infty} \mu\left\{\left(b_{1}\right)\right\} \leq \lim _{n \rightarrow \infty} \frac{2}{n}=0 .
$$

32. Portanto, $\mu\left(\left\{b_{1}\right\}\right)=0$, o que significa que a medida de um ponto é 0 , como havíamos afirmado.

33. $\log 0, \mu(P)=\mu\left(\bigcup_{i=1}^{n}\left\{a_{i}\right\}\right)=\mu\left(\left\{a_{1}\right\}\right)+\mu\left(\left\{a_{2}\right\}\right)+\ldots+\mu\left(\left\{a_{n}\right\}\right)=0$.

\section{Integral de Lebesgue e os Espaços $L^{p}$ 's}

34. Considere a medida de Lebesgue definida na $\sigma$-álgebra dos borelianos $\mathcal{B}$, dada em (24).

35. Lebesgue definiu uma integral que recebe o seu nome e generaliza a integral de Riemann no seguinte sentido: Toda função $f$ que é Riemann integrável (na definição da Análise Real [3, Capítulo 10]) é também Lebesgue integrável e as duas integrais são iguais. (Veja o Exercício 4.L em [2]).

36. Seja $\mathcal{B}$ a $\sigma$-álgebra do Borelianos e considere $\mu$ a medida de Lebesgue em $\mathcal{B}$. Se $\Omega \in \mathcal{B}$ e $f: \Omega \rightarrow \mathbb{R}$ é uma função mensurável, escrevemos a integral de Lebesgue de $f$ sobre $\Omega$ com o símbolo:

$$
\int_{\Omega} f d \mu \text {. }
$$


37. Embora tal integral possua uma definição precisa (e elegante, a propósito!), não a traremos aqui. O mais importante aqui é a relação entre as duas integrais, dada pelo teorema que se encontra em (35).

38. Em outras palavras, (35) diz que: Dados um intervalo $[a, b], a<b$, qualquer em $\mathcal{B}$ e $f:[a, b] \rightarrow \mathbb{R}$ uma função Riemann integrável, vale o seguinte resultado:

$$
\int_{a}^{b} f(x) d x=\int_{[a, b]} f d \mu .
$$

39. Isso significa que a integral de Lebesgue é uma extensão da integral de Riemann, ou seja, se $f$ é integrável segundo Riemann, então $f$ é integrável segundo Lebesgue.

40. Não vale a recíproca do item anterior, isto é, existem funções que são Lebesgue integráveis, mas não são Riemann integráveis.

41. Seja $f:[0,1] \rightarrow \mathbb{R}$ dada por

$$
f(x)=\left\{\begin{array}{ll}
1, & \text { se } x \notin \mathbb{Q} \\
0, & \text { se } x \in \mathbb{Q}
\end{array} .\right.
$$

A função $f$ é Lebesgue integrável, mas não é Riemann integrável.

42. Sejam $\Omega \subset \mathbb{R}$ um conjunto mensurável e $1 \leq p<\infty$. O espaço $L^{p}(\Omega)$ é definido como

$$
L^{p}(\Omega)=\left\{f: \Omega \rightarrow \mathbb{R} \text { mensurável : } \int_{\Omega}|f|^{p} d \mu<\infty\right\} .
$$

43. É possível mostrar que $L^{p}(\Omega)$ é um espaço vetorial normado.

44. Considere $\Omega=(1,+\infty)$ e $h(x)=\frac{1}{x^{2}}$, para $x \in \Omega$.

45. Como $h$ é contínua em seu domínio, segue de (19) que $h$ é mensurável.

46. Usando (38), temos

$$
\int_{(1,+\infty)} \frac{1}{x^{2}} d \mu=\int_{1}^{+\infty} \frac{1}{x^{2}} d x=\lim _{b \rightarrow+\infty}\left(-\left.\frac{1}{x}\right|_{1} ^{b}\right)=\lim _{b \rightarrow+\infty}\left[-\frac{1}{b}-\left(-\frac{1}{1}\right)\right]=1 .
$$


47. Portanto, $h \in L^{1}(\Omega)$, pois $\int_{(1,+\infty)}|h|^{1} d \mu<\infty$.

\section{Derivada Fraca}

48. Seja $I=(a, b), a<b$. O espaço $C_{0}^{1}(I)$ é o conjunto das funções $\psi: I \rightarrow \mathbb{R}$ tais que $\psi(a)=\psi(b)=0$ e $\psi \in C^{1}(I)$.

49. Relembrando, dizer que $\psi \in C^{1}(I)$ significa dizer que $\psi$ possui derivada contínua em $I$.

50. Temos que, se $f(x)=\operatorname{sen}(x), x \in(-\pi, \pi)$, então $f \in C_{0}^{1}(-\pi, \pi)$.

51. Considere a função $\eta:(-1,1) \rightarrow \mathbb{R}$ definida por

$$
\eta(t):=\left\{\begin{array}{cc}
\exp \left(\frac{1}{(2 t)^{2}-1}\right), & \text { se }|t|<1 / 2 \\
0, & \text { c.c. }
\end{array} .\right.
$$

52. É possível mostrar que $\eta \in C_{0}^{1}(-1,1)$. Mais do que isso, $\eta$ possui derivadas de todas as ordens, e estas se anulam em -1 e 1 .

53. Seja $u \in L^{p}(I)$, em que $I \subset \mathbb{R}$ é um intervalo e $1 \leq p<\infty$. Dizemos que $u$ é fracamente diferenciável ou diferenciável no sentido fraco se existir $v \in L^{p}(I)$ tal que

$$
\int_{I} u \phi^{\prime} d \mu=-\int_{I} v \phi d \mu, \forall \phi \in C_{0}^{1}(I) .
$$

54. Neste caso, chamamos $v$ de derivada fraca de $u$ e denotamos $v=u^{\prime}$.

55. Neste novo conceito, a função $u(x)=|x|, x \in(-1,1)$, possui derivada, e apresentamos a seguir a demonstração dessa afirmação.

56. Seja $I=(-1,1)$ e considere $u: I \rightarrow \mathbb{R}$ a função $u(x)=|x|$.

57. Como u é contínua, segue que u é mensurável.

58. Usando (38), não é difícil ver que $u \in L^{1}(I)$. 
59. Pela definição de módulo, $u(x)=\left\{\begin{array}{cc}x, & \text { se } x \geq 0 \\ -x, & \text { se } x<0\end{array}\right.$.

60. Assim, novamente por (38), $\forall \phi \in C_{0}^{1}(I)$,

$$
\int_{(-1,1)}|x| \phi^{\prime} d \mu=\int_{-1}^{1}|x| \phi^{\prime}(x) d x=\int_{-1}^{0}-x \phi^{\prime}(x) d x+\int_{0}^{1} x \phi^{\prime}(x) d x .
$$

61. Integrando por partes o lado direito da igualdade acima, obtém-se

$$
\begin{aligned}
\int_{-1}^{0}-x \phi^{\prime}(x) d x & =-\left\{\left.x \phi(x)\right|_{-1} ^{0}-\int_{-1}^{0} \phi(x) 1 d x\right\} \\
& =-[0 \phi(0)-(-1) \phi(-1)]-\int_{-1}^{0}-1 \phi(x) d x .
\end{aligned}
$$

62. Ora, $\forall \phi \in C_{0}^{1}(-1,1)$, vale $\phi(-1)=0$ e $\phi(1)=0$.

63. Daí e de (61),

$$
\begin{aligned}
\int_{-1}^{0}-x \phi^{\prime}(x) d x & =-[0 \cdot \phi(0)+1 \cdot 0]-\int_{-1}^{0}-1 \phi(x) d x \\
& =-\int_{-1}^{0}-1 \phi(x) d x .
\end{aligned}
$$

64. Analogamente,

$$
\begin{aligned}
\int_{0}^{1} x \phi^{\prime}(x) d x & =\left\{\left.x \phi(x)\right|_{0} ^{1}-\int_{0}^{1} 1 \phi(x) d x\right\} \\
& =[1 \phi(1)-0 \phi(0)]-\int_{0}^{1} 1 \phi(x) d x
\end{aligned}
$$

65. De (62) mais uma vez,

$$
\begin{aligned}
\int_{0}^{1} x \phi^{\prime}(x) d x & =[1 \cdot 0-0 \cdot \phi(0)]-\int_{0}^{1} 1 \phi(x) d x \\
& =-\int_{0}^{1} 1 \phi(x) d x .
\end{aligned}
$$

66. Portanto, de (60), 63) e 665), temos

$$
\int_{-1}^{1}|x| \phi^{\prime}(x) d x=-\int_{-1}^{0}-1 \phi(x) d x-\int_{0}^{1} 1 \phi(x) d x .
$$


67. Logo, a derivada fraca de $u$ é dada por

$$
v(x)=\left\{\begin{array}{cc}
-1, & \text { se }-1<x \leq 0 \\
1, & \text { se } 0<x<1
\end{array},\right.
$$

uma vez que a definição em (53) é satisfeita, isto é, $\forall \phi \in C_{0}^{1}(-1,1)$,

$$
\int_{-1}^{1}|x| \phi^{\prime}(x) d x=-\int_{-1}^{1} v(x) \phi(x) d x .
$$

68. Conclui-se que a função módulo, que não tem derivada clássica, possui derivada fraca, dada em (67).

\section{Considerações Finais}

69. Toda função diferenciável no sentido clássico do Cálculo possui derivada fraca, e ambas são iguais. Portanto, a derivada fraca de $f(x)=x^{3}$, $x \in(-5,5)$, por exemplo, é $f^{\prime}(x)=3 x^{2}$.

70. Uma função pode não ser derivável no sentido clássico nem no sentido fraco.

\section{Agradecimentos}

Agradecemos ao Programa Institucional Voluntário de Iniciação Científica, da Universidade Federal do Tocantins, pela oportunidade de participar do PIVIC. Esse trabalho faz parte do primeiro trimestre da Iniciação Científica, cuja pesquisa tem como tema os Espaços de Sobolev e Métodos Variacionais para resolução de EDO's.

\section{Ciência Aberta}

$\mathrm{O}$ arquivo latex para este artigo, juntamente com outros arquivos suplementares, estão disponíveis [4]. 


\section{Referências}

[1] Brezis, H. Functional Analysis, Sobolev Spaces and Partial Differential Equations. New York, Springer, 2010.

[2] Bartle, Robert L. The Elements of Integration and Lebesgue Measure. John Wiley \& Sons, 1995.

[3] Lima, E. L. Análise Real. Volume 1, Rio de Janeiro, IMPA, Coleção Universitária, 1989.

[4] Lobo, Matheus P. "Open Journal of Mathematics and Physics (OJMP)." OSF, 21 Apr. 2020.

https://doi.org/10.17605/osf.io/6hzyp 


\section{Colaboração Matemática Aberta}

Thafne Sirqueira Carvalho ${ }^{1}$

(autor principal, thafne.sirqueira@uft.edu.br)

https://orcid.org/0000-0001-6275-771X

José Carlos de Oliveira Junior ${ }^{1}$

https://orcid.org/0000-0002-6518-5566

${ }^{1}$ Universidade Federal do Tocantins (Brasil) 